Proceedings of the Edinburgh Mathematical Society (2004) 47, 35-51 (C)

DOI:10.1017/S001309150200113X Printed in the United Kingdom

\title{
AN ANALOGUE OF CIRCULAR UNITS FOR PRODUCTS OF ELLIPTIC CURVES
}

\author{
SRINATH BABA ${ }^{1 *}$ AND RAMESH SREEKANTAN ${ }^{2} \dagger$ \\ ${ }^{1}$ Department of Mathematics, McGill University, Montreal, \\ Quebec H3A 2K6, Canada (sbaba@math.mcgill.ca) \\ ${ }^{2}$ School of Mathematics, Tata Institute of Fundamental Research, \\ Colaba, Mumbai 400 005, India (ramesh@math.tifr.res.in)
}

(Received 4 December 2002)

Dedicated to the memory of Rob Alasdair Mac Fhraing (1915-2001)

\begin{abstract}
We construct certain elements in the motivic cohomology group $H_{\mathcal{M}}^{3}\left(E \times E^{\prime}, \mathbb{Q}(2)\right)$, where $E$ and $E^{\prime}$ are elliptic curves over $\mathbb{Q}$. When $E$ is not isogenous to $E^{\prime}$ these elements are analogous to circular units in real quadratic fields, as they come from modular parametrizations of the elliptic curves. We then find an analogue of the class-number formula for real quadratic fields, which specializes to the usual quadratic class-number formula when $E$ and $E^{\prime}$ are quadratic twists.
\end{abstract}

Keywords: motivic cohomology; class number; Beilinson conjectures

2000 Mathematics subject classification: Primary 11F67; 14G35

Secondary 11F11; 11E45; 14G10

\section{Introduction}

The classical formulae of Dirichlet and Dedekind give interpretations to the special values of the zeta functions of number fields. For example, one has

$$
\zeta_{K}^{*}(0)=\frac{-h_{K} R_{K}}{W_{K}}
$$

where $\zeta_{K}^{*}(0)$ is the first non-zero term in the Taylor expansion around $s=0$ of the zeta function $\zeta_{K}(s), h_{k}$ is the class number, $R_{K}$ is the regulator, and $W_{K}$ is the number of roots of unity in $K$.

In some special cases, one has a more precise expression for the value of the zeta function. For example, if $K$ is a real quadratic field and $\chi$ is its quadratic character of conductor $N$, one has

$$
L^{\prime}(0, \chi)=\log \left|U_{\chi}\right|
$$

* Present address: Max Planck Institute of Mathematics, Vivatsgasse 7, D-53111 Bonn, Germany.

$\dagger$ Present address: University of Toronto, Toronto, Ontario M5S 1A1, Canada (ramesh@math. toronto.edu). 
where $\xi=\mathrm{e}^{2 \pi \mathrm{i} / N}$ and

$$
U_{\chi}=\prod_{\substack{k \bmod N \\(k, N)=1}}\left(1-\xi^{k}\right)^{-\chi(k) / 2}=N_{K}^{\mathbb{Q}(\xi)}\left(\sqrt{\frac{1-\xi^{b}}{1-\xi}}\right)
$$

is the norm of a cyclotomic unit called a circular unit. Here $b$ is any non-square modulo $N$. This gives the formula

$$
L^{\prime}(0, \chi)=\log \prod_{\substack{k \bmod N \\(k, N)=1}}\left|1-\xi^{k}\right|^{-\chi(k) / 2}=\sum_{\substack{k \bmod N \\(k, N)=1}}-\frac{1}{2} \chi(k) \log \left|1-\xi^{k}\right| .
$$

Beilinson [2], expanding on the work of others, formulated conjectures which generalized part of (1.1). His conjectures give an interpretation of the special values of the $L$-functions of an algebraic variety in terms of certain groups associated to the variety. In particular, Beilinson made the following generalization of the Tate conjecture.

Conjecture 1.1 (Beilinson). Let $X$ be a smooth projective variety defined over $\mathbb{Q}$ and let $m$ be an integer. Let $L\left(H^{2 m}(X), s\right)$ denote the $L$-function defined by $H^{2 m}(X)$ and let $B^{m}(X)_{\mathbb{Q}}$ denote the $\mathbb{Q}$-vector space generated by the codimensional $m$-cycles modulo homological equivalence. Then

(i) $\tilde{r}_{\mathcal{D}}\left(B^{m}(X)_{\mathbb{Q}} \oplus H_{\mathcal{M}}^{2 m+1}(X, \mathbb{Q}(m+1))_{\mathbb{Z}}\right)$ induces a $\mathbb{Q}$-structure on

$$
H_{\mathcal{D}}^{2 m+1}\left(X_{/ \mathbb{R}}, \mathbb{R}(m+1)\right)
$$

(ii) $\operatorname{ord}_{s=m} L\left(H^{2 m}(X), s\right)=\operatorname{dim}_{\mathbb{Q}} H_{\mathcal{M}}^{2 m+1}(X, \mathbb{Q}(m+1))_{\mathbb{Z}}$;

(iii) $\operatorname{ord}_{s=m+1} L\left(H^{2 m}(X), s\right)=-\operatorname{dim}_{\mathbb{Q}}\left(B^{m}(X)_{\mathbb{Q}}\right)$ (Tate);

(iv) $L^{*}\left(H^{2 m}(X), s\right)_{s=m} \sim_{\mathbb{Q}^{*}} c_{X}(m)$.

Here $\tilde{r}_{\mathcal{D}}$ is a certain 'thickened' regulator map which generalizes the usual regulator map for number fields, $H_{\mathcal{M}}^{2 m+1}(X, \mathbb{Q}(m+1))_{\mathbb{Z}}$ is the 'integral' motivic cohomology, which is the motivic cohomology of a regular proper model, if it exists. Otherwise there is an unconditional definition due to Scholl $[\mathbf{1 2}]$, and $H_{\mathcal{D}}^{2 m+1}\left(X_{/ \mathbb{R}}, \mathbb{R}(m+1)\right)$ is the 'real' Deligne cohomology which is a real vector space of dimension

$$
\underset{s=m}{\operatorname{ord}} L\left(H^{2 m}(X), s\right)-\underset{s=m+1}{\operatorname{ord}} L\left(H^{2 m}(X), s\right)
$$

over $\mathbb{R}$. Here $L^{*}\left(H^{2 m}(X), s\right)_{s=m}$ is the first non-zero term in the Taylor expansion and $c_{X}(m)$ is an element of $\mathbb{R}^{*} / \mathbb{Q}^{*}$ related to the covolume of the image of $\tilde{r}_{\mathcal{D}}$. More generally, the conjecture can be stated for motives - namely individual pieces of the cohomology group which are, in particular, Galois invariant. A good reference for the background behind the conjectures is the paper of Schneider $[\mathbf{1 1}]$.

If $K$ is a number field and $X=\operatorname{Spec}(K)$ and $m=0$, we recover the classical results. Beilinson proved his conjectures in some special cases, including that of the product of two modular curves and $m=1$, from which they follow for the case of the product of two Elliptic curves over $\mathbb{Q}$. The aim of this paper is to show that formulae analogous to $(1.2)$ and (1.3) also exist. 
Theorem 1.2. Let $E$ and $E^{\prime}$ be non-isogenous elliptic curves over $\mathbb{Q}$ corresponding to modular forms $f$ and $g$ of level $N_{1}$ and $N_{2}$, respectively. Let $M=\operatorname{gcd}\left(N_{1}, N_{2}\right)$ and $N=\operatorname{lcm}\left(N_{1}, N_{2}\right)$. Assume $N / M \neq 1$. There is an element $\Psi_{*}\left(\Xi_{0}\left(N_{1}, N_{2}\right)\right)$ in $H_{\mathcal{M}}^{3}\left(E \times E^{\prime}, \mathbb{Q}(2)\right)$, an analogue of a circular unit, such that

$$
L^{\prime}\left(H^{1}(E) \otimes H^{1}\left(E^{\prime}\right), 1\right)=\frac{-\operatorname{deg}(\psi)}{12 c(\phi) c\left(\phi^{\prime}\right)\left[\Gamma: \Gamma_{0}(N / M)\right]}\left\langle r_{\mathcal{D}}\left(\psi_{*}\left(\Xi_{0}\left(N_{1}, N_{2}\right)\right)\right), \omega_{E} \wedge \bar{\omega}_{E^{\prime}}\right\rangle,
$$

where $\psi$ is the map from $X_{0}(N) \times X_{0}(N)$ to $E \times E^{\prime}$ and $c(\phi), c\left(\phi^{\prime}\right)$ are the constants coming from the modular parametrization.

One also has an analogue of (1.3). Let

$$
\log _{q}(t)=\frac{1}{24} \log |q t|+\sum_{n=1}^{\infty} \log \left|1-q^{n} t\right|
$$

where $q=\mathrm{e}^{2 \pi \mathrm{i} z}$.

Theorem 1.3. Let $E, E^{\prime}$ be non-isogenous elliptic curves over $\mathbb{Q}$ corresponding to modular forms $f$ and $g$ of level $N_{1}$ and $N_{2}$, respectively, and let $\xi$ be a primitive $(N / M)$ th root of unity, where $N=\operatorname{lcm}\left(N_{1}, N_{2}\right)$ and $M=\operatorname{gcd}\left(N_{1}, N_{2}\right)$. Then

$$
L^{\prime}\left(H^{1}(E) \otimes H^{1}\left(E^{\prime}\right), 1\right)=-\frac{1}{2} \sum_{\substack{k \bmod (N / M) \\(k, N / M)=1}} \frac{1}{2 \pi \mathrm{i}} \int_{X_{0}(N)} \log _{q}\left(\xi^{k}\right) f(q) \overline{g(q)} \frac{\mathrm{d} q}{q} \frac{\mathrm{d} \bar{q}}{\bar{q}} .
$$

Furthermore, one can find elements of $H_{\mathcal{M}}^{3}\left(X_{1}(N) \times X_{1}(N), \mathbb{Q}(2)\right)$ such that the integral can be interpreted as the regulator of those elements. These elements are analogues of cyclotomic units.

In fact there appears to be a correspondence between quadratic fields and products of elliptic curves with the product of non-isogenous elliptic curves behaving like real quadratic fields and isogenous elliptic curves behaving like imaginary quadratic fields. In the last section we have a table which illustrates this correspondence.

The proof follows by looking at Ogg's [10] original proof of the Tate conjecture for products of two elliptic curves more carefully and using Kronecker's first limit formula. We also show that we can recover the usual class-number formula (1.3) by specializing to the case of the product $E \times E_{\chi}$, where $E_{\chi}$ is a real quadratic twist of $E$.

\section{Part I: special values of $L$-functions}

\subsection{Preliminaries}

Let $E$ and $E^{\prime}$ be the two elliptic curves over $\mathbb{Q}$. Let $\omega_{E}$ and $\omega_{E^{\prime}}$ be Néron differentials corresponding to the global minimal Weierstrass models. These are defined up to \pm 1 . Let $f$ and $g$ be the Hecke eigenforms of weight 2 of levels $N_{1}$ and $N_{2}$, corresponding to $E$ and $E^{\prime}$, respectively. We can assume that they are normalized newforms of levels $N_{1}$ and $N_{2}$, respectively, and we will assume that they are square free. 
Let $N=\operatorname{lcm}\left(N_{1}, N_{2}\right)$ and let $M=\operatorname{gcd}\left(N_{1}, N_{2}\right)$. We will think of $f$ and $g$ as modular forms for $\Gamma_{0}(N)$. Let $\phi$ and $\phi^{\prime}$ be the modular parametrizations from $X_{0}(N)$ to $E$ and $E^{\prime}$, respectively. Define $c(\phi)$ and $c\left(\phi^{\prime}\right)$ in $\mathbb{Q}^{*}$ by

$$
\phi^{*}\left(\omega_{E}\right)=c(\phi) 2 \pi \mathrm{i} f(z) \mathrm{d} z \quad \text { and } \quad \phi^{\prime *}\left(\omega_{E^{\prime}}\right)=c\left(\phi^{\prime}\right) 2 \pi \mathrm{i} g(z) \mathrm{d} z,
$$

where by i we denote a choice of a $\sqrt{-1}$ that we make once and for all. It turns out that $c(\phi)$ and $c\left(\phi^{\prime}\right)$ are actually in $\mathbb{Z} \backslash\{0\}$.

Let $X_{0}(N)$ denote the modular curve with level $N$ structure. This is an algebraic curve defined over $\mathbb{Q}$ whose complex points can be identified with the compactification of the fundamental domain for $\Gamma_{0}(N)$. On occasion we will also use this to represent the complex manifold $X_{0}(N)(\mathbb{C})$. Let

$$
f(z)=\sum_{n=1}^{\infty} a_{n} q^{n} \quad \text { and } \quad g(z)=\sum_{n=1}^{\infty} b_{n} q^{n}
$$

be the Fourier expansions of $f$ and $g$. Define

$$
\delta(f, g):=f(z) \overline{g(z)} \mathrm{d} x \mathrm{~d} y=\frac{1}{2} \mathrm{i} f(z) \overline{g(z)} \mathrm{d} z \mathrm{~d} \bar{z},
$$

where $z=x+\mathrm{i} y$. Define the Petersson inner product $(f, g)$ by

$$
(f, g)=\frac{1}{\left[\Gamma: \Gamma_{0}(N)\right]} \int_{X_{0}(N)} \delta(f, g) .
$$

We will use the following two theorems of Ogg [10].

Theorem 2.1 (Ogg). If $f$ and $g$ are normalized of levels $N_{1}$ and $N_{2}$, respectively, and $(f, g) \neq 0$, then $f=g$ (and $\left.N_{1}=N_{2}\right)$.

Theorem $2.2(\mathrm{Ogg})$. If $f=\sum_{n=1}^{\infty} a_{n} q^{n}$ is a normalized cusp form of square-free level $N$ and $p \mid N$, then $a_{p}= \pm 1$.

Let $L\left(H^{2}\left(E \times E^{\prime}\right), s\right)$ be the $L$-function of the product of the two elliptic curves. Then one has

$$
L\left(H^{2}\left(E \times E^{\prime}\right), s\right)=\zeta(s-1)^{2} A(s-1) L_{f, g}(s-1),
$$

where $\zeta(s)$ is the Riemann zeta function, $\zeta_{N}(s)$ is the same function with the primes dividing $N$ removed,

$$
L_{f, g}(s)=\zeta_{N}(2 s) \sum_{n=1}^{\infty} \frac{a_{n} \bar{b}_{n}}{n^{s+1}},
$$

and $A(s)$ is a term depending on primes dividing $N$, related to the $L$-factor at the primes of bad reduction. In general these factors can be quite complicated, but Ogg [10] proves a functional equation in a special case. This suggests that the $L$-factors should be as follows.

If $p \mid M$, then the $L$-factor is

$$
\frac{1}{\left(1-a_{p} b_{p} p^{-s}\right)\left(1-a_{p} b_{p} p^{-(s+1)}\right)},
$$


while if $p \mid(N / M)$, it is

$$
\frac{1}{\left(1-a_{p} b_{p} p^{-(s+1)}+p^{-1-2 s}\right)},
$$

at least when $E$ has multiplicative reduction at $p$. Recall that if $p \mid N, a_{p}$ is 0,1 or -1 depending on whether the reduction is additive, split multiplicative or non-split multiplicative. Since we have assumed that the levels are square free, all the reduction is multiplicative.

Remark 2.3. Since $b_{n}$ is in $\mathbb{Q}$, writing $\bar{b}_{n}$ is redundant, but, as in general it is the complex conjugate that appears in the Rankin-Selberg convolution of two modular forms, we have chosen to leave it in.

\subsection{The Rankin-Selberg convolution}

Using the Rankin-Selberg convolution [10] we get the following integral representation of $L_{f, g}(s)$ :

$$
\Phi(s):=\left(\frac{2 \pi}{\sqrt{N}}\right)^{-2 s} \Gamma(s) \Gamma(s+1) L_{f, g}(s)=2 \pi \sum_{d \mid N} \frac{\mu(d)}{d^{s}} \int_{X_{0}(N)} \delta(f, g) E_{\infty}^{*}\left(\frac{N z}{d}, s\right),
$$

where $E_{\infty}^{*}(z, s)$ is the Eisenstein-Kronecker-Lerch series or the Epstein zeta function:

$$
E_{\infty}^{*}(z, s)=\left(\frac{1}{\pi}\right)^{s} \Gamma(s) \sum_{\gamma \in \Gamma / \Gamma_{\infty}} \operatorname{Im}(\gamma z)^{s}=\left(\frac{1}{\pi}\right)^{s} \Gamma(s) \sum_{m, n}^{\prime} \frac{y^{s}}{|m z+n|^{2 s}} .
$$

It converges for $\operatorname{Re}(s)>1$, has a meromorphic continuation to the entire complex plane and satisfies the functional equation

$$
E_{\infty}^{*}(z, s)=E_{\infty}^{*}(z, 1-s)
$$

Furthermore, it has a simple pole with residue 1 at $s=1$ independent of $z$. A good reference for all these facts is Lang's book Elliptic functions [6].

From this one can see that the residue of $\Phi(s)$ at $s=1$ is a constant multiplied by $(f, g)$ and one has the following.

Theorem 2.4 (Rankin). The function

$$
L_{f, g}(s)=\zeta_{N}(2 s) \sum_{n=1}^{\infty} a_{n} \bar{b}_{n} n^{-(s+1)}
$$

is entire if $(f, g)=0$ and is entire except for a simple pole at $s=1$ if $(f, g) \neq 0$. In this case the residue is a rational multiple of $(f, g)$.

Using this, the following theorem holds.

Theorem 2.5 (Ogg). We have $L_{f, g}(1) \neq 0$ if $(f, g)=0$.

Using these results and an observation on the non-vanishing of $A(1)$, Ogg [10] proved the Tate conjecture. 


\subsubsection{Functional equation}

We are interested in the value of $L^{*}\left(H^{1}(E) \otimes H^{1}\left(E^{\prime}\right), s\right)$ at $s=1$. For this we need the functional equation for $L_{f, g}(s)$.

Theorem 2.6 (Ogg). Let $f$ and $g$ be normalized cusp forms of square-free level $N_{1}$, $N_{2}$. Let $N=\operatorname{lcm}\left(N_{1}, N_{2}\right)$ and $M=\operatorname{gcd}\left(N_{1}, N_{2}\right)$. Recall

$$
\Phi(s):=\left(\frac{2 \pi}{\sqrt{N}}\right)^{-2 s} \Gamma(s) \Gamma(s+1) L_{f, g}(s)
$$

and set

$$
\Phi^{+}(s)=\Phi(s) A(s)
$$

where

$$
A(s)=\prod_{p \mid M}\left(1-a_{p} b_{p} p^{-s}\right)^{-1}
$$

Then

$$
\Phi^{+}(s)=\Phi^{+}(1-s) .
$$

Furthermore, one has the following expression for $\Phi^{+}(s)$ :

$$
\Phi^{+}(s)=2 \pi \sum_{d \mid(N / M)} \frac{\mu(d)}{d^{s}} \int_{X_{0}(N)} E_{\infty}^{*}\left(\frac{N z}{d}, s\right) \delta(f, g) .
$$

Proof. The formula (2.5) is immediate from the proof of the theorem in [10].

Since $\Phi^{+}(s)$ satisfies a simple functional equation, it is a good candidate for the completed zeta function of $L\left(H^{1}(E) \otimes H^{1}\left(E^{\prime}\right), s+1\right)$. From this one can guess the local factors at the primes of bad reduction. A generalization of this theorem for arbitrary $N$ was proved in [7].

From this point on we will treat $\Phi^{+}(s-1)$ as the completed zeta function of $L\left(H^{1}(E) \otimes\right.$ $\left.H^{1}\left(E^{\prime}\right), s\right)$ and we will consider three cases, namely, when $E$ is isogenous to $E^{\prime}$, when $E$ is not isogenous to $E^{\prime}$ over $\overline{\mathbb{Q}}$, and, finally, when $E$ is not isogenous to $E^{\prime}$ over $\mathbb{Q}$, but is isogenous over some finite, necessarily quadratic, extension. In the third case the level is no longer square free but we can still handle it.

\section{3. $E$ is isogenous to $E^{\prime}$}

In this case $N_{1}=N_{2}=N, f=g$ and $N / M=1$. From Rankin's Theorem, $L_{f, g}(s)=$ $L_{f, f}(s)$ has a pole at $s=1$ and, from the motivic decomposition, we have

$$
L\left(H^{1}(E) \otimes H^{1}(E), s\right)=L\left(H^{2}(E), s\right) L\left(\operatorname{Sym}^{2}(E), s\right) .
$$

So, from (2.1) above, we have that

$$
L\left(\operatorname{Sym}^{2}(E), s\right)=\frac{N^{-(s-1)} \Phi^{+}(s-1)}{(2 \pi)^{-2(s-1)} \Gamma(s-1) \Gamma(s) \zeta(s-1)},
$$


and, using the integral expression (2.5), that

$$
\Phi^{+}(0)=\lim _{s \rightarrow 0} 2 \pi \int_{X_{0}(N)} \delta(f, f) E_{\infty}^{*}(N z, s)
$$

Since $E_{\infty}^{*}(N z, 0)$ has a simple pole with residue 1 at $s=0$ independent of $z$ and $\zeta(0)=-\frac{1}{2}$ we have

$$
L\left(\operatorname{Sym}^{2}(E), 1\right)=-4 \pi \lim _{s \rightarrow 0} \frac{1}{\Gamma(s)} \int_{X_{0}(N)} \delta(f, f) E_{\infty}^{*}(N z, s)=-4 \pi\left[\Gamma: \Gamma_{0}(N)\right](f, f) .
$$

From the relation between the canonical differential and the modular form one has

$$
\left[\Gamma: \Gamma_{0}(N)\right](f, f)=\frac{\mathrm{i} \operatorname{deg}(\phi)}{8 \pi^{2} c(\phi)^{2}} \int_{E(\mathbb{C})} \omega_{E} \wedge \bar{\omega}_{E^{\prime}}
$$

Using this in (2.7) one gets

$$
L\left(\operatorname{Sym}^{2} E, 1\right)=\frac{\operatorname{deg}(\phi)}{c(\phi)^{2}} \frac{1}{2 \pi \mathrm{i}} \int_{E(\mathbb{C})} \omega_{E} \wedge \bar{\omega}_{E^{\prime}}
$$

Remark 2.7. From [4] we have that the constant

$$
c_{\operatorname{Sym}^{2}(E)}(1)=\frac{1}{2 \pi \mathrm{i}} \int_{E(\mathbb{C})} \omega_{E} \wedge \bar{\omega}_{E^{\prime}},
$$

so part (iv) of Beilinson's conjecture follows from this calculation.

\section{4. $E$ is not isogenous to $E^{\prime}$ over $\overline{\mathbb{Q}}$}

In this case $N_{1}$ and $N_{2}$ are square free and $N / M$ is the product of the primes dividing $N_{1}$ or $N_{2}$, but not both. We make the added assumption that $N / M \neq 1$. The expression for $\Phi^{+}(s)$ and the fact that $L_{f, g}(s)$ has a simple zero at $s=0$ show that $L\left(H^{1}(E) \otimes\right.$ $\left.H^{1}\left(E^{\prime}\right), s\right)$ has a simple zero at $s=1$ and $L^{\prime}\left(H^{1}(E) \otimes H^{1}\left(E^{\prime}\right), 1\right)=\Phi^{+}(0)$.

To compute $\Phi^{+}(0)$ from (2.5), we need to understand the behaviour of the Eisenstein series $E_{\infty}^{*}(z, s)$ as $s$ approaches 0. For this we need Kronecker's First Limit Formula.

Theorem 2.8 (Kronecker). Let

$$
E_{\infty}^{*}(z, s)=\pi^{-s} \Gamma(s) \sum_{m, n}^{\prime} \frac{y^{s}}{|m z+n|^{2 s}}
$$

and

$$
\eta(z)=q^{1 / 24} \prod_{n=1}^{\infty}\left(1-q^{n}\right)
$$

where $q=\mathrm{e}^{2 \pi \mathrm{i} z}$, and let $\gamma$ be Euler's constant. Then

$$
E_{\infty}^{*}(z, s)=\Gamma(s) \pi^{-s}\left(\frac{\pi}{s-1}-\log y+2 \pi(\gamma-\log 2)-4 \pi \log |\eta(z)|+O(s-1)\right) .
$$


Proof. The proof of this theorem can be found in Lang [6, p. 273].

Using the limit formula, we get an expression for the special value $\Phi^{+}(0)$. Let $\Delta(z)$ be the usual cusp form of weight 12 for $S L_{2}(\mathbb{Z})$. We have $\Delta(z)=\eta(z)^{24}$. Define, for $N=\operatorname{lcm}\left(N_{1}, N_{2}\right)$ and $M=\operatorname{gcd}\left(N_{1}, N_{2}\right)$ as before,

$$
\Delta_{N_{1}, N_{2}}(z):=\prod_{d \mid(N / M)} \Delta\left(\frac{N z}{d}\right)^{\mu(d)} .
$$

This is a modular unit as $\sum_{d \mid(N / M)} \mu(d)=0$ and its divisor is supported on the cusps of $X_{0}(N)$.

Theorem 2.9. Let $f, g, N$ and $M$ be as above. Then

$$
\Phi^{+}(0)=-\frac{1}{3} \pi \int_{X_{0}(N)} \log \left|\Delta_{N_{1}, N_{2}}(z)\right| \delta(f, g) .
$$

Proof. From the functional equation, we have

$$
E_{\infty}^{*}\left(\frac{N z}{d}, s\right)=E_{\infty}^{*}\left(\frac{N z}{d}, 1-s\right)
$$

Combining this with the limit formula (2.9), we have

$$
\begin{aligned}
\lim _{s \rightarrow 0} E_{\infty}^{*}\left(\frac{N z}{d}, s\right) & =\lim _{s \rightarrow 0} E_{\infty}^{*}\left(\frac{N z}{d}, 1-s\right) \\
& =\frac{1}{-s}-\log \frac{N y}{d}+2(\gamma-\log 2)-4 \log \left|\eta\left(\frac{N z}{d}\right)\right|+O(-s) .
\end{aligned}
$$

Using result (2.5) we have

$$
\Phi^{+}(0)=2 \pi \int_{X_{0}(N)} \sum_{d \mid(N / M)} \mu(d)\left(\frac{1}{-s}-\log \frac{N y}{d}+2(\gamma-\log 2)-4 \log \left|\eta\left(\frac{N z}{d}\right)\right|\right) \delta(f, g) .
$$

This simplifies to

$$
\Phi^{+}(0)=2 \pi \int_{X_{0}(N)} \sum_{d \mid(N / M)} \mu(d)\left(-4 \log \left|\eta\left(\frac{N z}{d}\right)\right|\right) \delta(f, g)
$$

as $(f, g)=0$, and by assumption $(N / M)>1$ so $\sum_{d \mid(N / M)} \mu(d)=0$ and

$$
\prod_{d \mid(N / M)}\left(\frac{N}{d}\right)^{\mu(d)}=\prod_{d \mid(N / M)}\left(\frac{N}{M d}\right)^{\mu(d)} M^{\mu(d)}=1 .
$$

From the expression for $\Delta_{N_{1}, N_{2}}(z)$ we see that the Eisenstein series tends to

$$
\frac{1}{8} \log \left|\Delta_{N_{1}, N_{2}}(z)\right|
$$


and one has

$$
\Phi^{+}(0)=-\frac{1}{3} \pi \int_{X_{0}(N)} \log \left|\Delta_{N_{1}, N_{2}}(z)\right| \delta(f, g)
$$

Let

$$
\psi: X_{0}(N) \times X_{0}(N) \rightarrow E \times E^{\prime}
$$

be induced by the modular parametrizations $\phi$ and $\phi^{\prime}$ and let $\omega_{E}$ and $\omega_{E^{\prime}}$ be the canonical differentials on $E$ and $E^{\prime}$, respectively. Then one has $\phi^{*}\left(\omega_{E}\right)=2 \pi \mathrm{i} c(\phi) f(z) \mathrm{d} z$ and $\phi^{\prime *}\left(\omega_{E^{\prime}}\right)=2 \pi \mathrm{i} c\left(\phi^{\prime}\right) g(z) \mathrm{d} z$, giving

$$
\delta(f, g)=\frac{\operatorname{deg}(\psi)}{8 \pi^{2} \mathrm{i} c(\phi) c\left(\phi^{\prime}\right)} \omega_{E} \wedge \bar{\omega}_{E^{\prime}}
$$

Here $\operatorname{deg}(\psi)$ denotes the degree of the map $\left.\psi\right|_{\text {diagonal }}$.

Therefore, one has

$$
\begin{aligned}
L^{*}\left(H^{1}(E)\right. & \left.\otimes H^{1}\left(E^{\prime}\right), 1\right) \\
= & \Phi^{+}(0) \\
= & -\frac{1}{3} \pi \int_{X_{0}(N)} \log \left|\Delta_{N_{1}, N_{2}}(z)\right| \delta(f, g) \\
= & -\frac{2}{3} \pi^{2} \mathrm{i} \frac{1}{2 \pi \mathrm{i}} \int_{X_{0}(N)} \log \left|\Delta_{N_{1}, N_{2}}(z)\right| \delta(f, g) \\
= & -\frac{2}{3} \pi^{2} \mathrm{i} \frac{1}{2 \pi \mathrm{i}} \int_{\psi_{*}\left(X_{0}(N)\right)} \log \left|\psi_{*}\left(\Delta_{N_{1}, N_{2}}(z)\right)\right| \frac{\operatorname{deg}(\psi)}{8 \pi^{2} \mathrm{i} c(\phi) c\left(\phi^{\prime}\right)} \omega_{E} \wedge \bar{\omega}_{E^{\prime}} \\
= & \frac{-\operatorname{deg}(\psi)}{12 c(\phi) c\left(\phi^{\prime}\right)} \frac{1}{2 \pi \mathrm{i}} \int_{\psi_{*}\left(X_{0}(N)\right)} \log \left|\psi_{*}\left(\Delta_{N_{1}, N_{2}}(z)\right)\right| \omega_{E} \wedge \bar{\omega}_{E^{\prime}} .
\end{aligned}
$$

\section{Part II: elements of $K$-theory and regulators}

Now we show that the special value of the $L$-function (2.16) can be interpreted as the regulator of explicit elements of the motivic cohomology of the product of the two elliptic curves. We will also show a formula analogous to the class-number formula for quadratic fields.

\subsection{Elements of $H_{\mathcal{M}}^{3}(X, \mathbb{Q}(2))$}

Let $X$ be a surface defined over $\mathbb{Q}$. The group $H_{\mathcal{M}}^{3}(X, \mathbb{Q}(2))$ has several different descriptions: first, in terms of a graded piece of $K_{1}(X)$; second, as the higher Chow group $C H^{2}(X, 1)$; and, finally, as the $K$-cohomology group $H^{1}\left(X, \mathcal{K}_{2}\right)$. From the third description and the Gersten-Quillen resolution, an element of the group is represented by a formal sum $\sum(\mathcal{C}, f)$, where $\mathcal{C}$ are curves on $X$ and $f$ are functions on these curves subject to the cocycle condition

$$
\sum \operatorname{div}(f)=0
$$


This is a generalization of the fact that elements of $F^{*}$ are elements of $K_{1}$ of a number field $F$.

\subsection{Products of modular curves}

Let $N$ be a square-free integer. In this section we will use the function

$$
\Delta_{N}(z):=\prod_{d \mid N} \Delta\left(\frac{N z}{d}\right)^{\mu(d)}
$$

on $X_{0}(N)$ to construct an element of $H_{\mathcal{M}}^{3}\left(X_{0}(N) \times X_{0}(N), \mathbb{Q}(2)\right)$ of the sort described above, using the diagonal embedding of $X_{0}(N)$ into $X_{0}(N) \times X_{0}(N)$.

Since $N$ is square free, the cusps are indexed by the set of divisors. Let $P_{d}$ denote the cusp represented by the class of $1 / d$. From [8] (though such calculations can also be found in [9]), one has that, for $N^{\prime} \mid N$,

$$
\underset{P_{d}}{\operatorname{ord}}\left(\Delta\left(N^{\prime}\right)\right)=N \frac{\operatorname{gcd}\left(d, N^{\prime}\right)}{\operatorname{lcm}\left(d, N^{\prime}\right)}
$$

where by $\Delta(k)$ we mean the function $\Delta(k z)$. Using this we can compute the orders of various products and quotients of the $\Delta$ function. In particular,

$$
\operatorname{div}\left(\Delta_{N}\right)=\prod_{p \mid N}(p-1)\left(\sum_{d \mid N} \mu(N / d) P_{d}\right) .
$$

Define a simple unit to be a modular unit with divisor of the form $k(P-Q)$ for some cusps $P$ and $Q$ and natural number $k$. From the Manin-Drinfel'd Theorem, we know that there is a number $\kappa$ such that $\Delta_{N}^{\kappa}$ can be decomposed as a product of simple units. However, for our purposes, we need a more explicit description.

Theorem 3.1. Let

$$
N=\prod_{i=0}^{r} p_{i} \quad \text { and } \quad \kappa=\prod_{i=1}^{r}\left(p_{i}+1\right) .
$$

The function $\Delta_{N}^{\kappa}$ is given by

$$
\Delta_{N}^{\kappa}=\prod_{d \mid\left(N / p_{0}\right)} F_{d}
$$

where $F_{d}$ are simple units with divisors

$$
\operatorname{div}\left(F_{d}\right)=\left(p_{0}-1\right) \mu(N / d) \prod_{i=1}^{r}\left(p_{i}^{2}-1\right)\left(P_{d}-P_{d p_{0}}\right) .
$$

Proof. The proof is algorithmic. Let $p_{0}$ be a prime dividing $N$. If $N=p_{0}$, then $\Delta_{N}(z)=\Delta\left(p_{0} z\right) / \Delta(z)$, there are two cusps $P_{1}$ and $P_{p_{0}}$ and one has

$$
\operatorname{div}\left(\Delta_{p_{0}}\right)=\left(p_{0}-1\right)\left(P_{p_{0}}-P_{1}\right) .
$$


In general, suppose that $q \neq p_{0}$ is a prime dividing $N$. The set of cusps can be partitioned into two sets of equal cardinality, one corresponding to $d \mid N$ with $(d, q)=1$ and the other corresponding to $d \mid N$ with $(d, q)=q$.

Now consider the product

$$
\prod_{d \mid(N / q)}\left(\frac{\Delta(q d z)^{q}}{\Delta(d z)}\right)^{\mu(N / q d)}=\prod f_{d}(z)^{\mu(N / q d)}
$$

where $f_{d}(z)=\Delta(q d z)^{q} / \Delta(d z)$.

We claim that this function has a divisor supported on only those cusps $P_{d^{\prime}}$ with $q \mid d^{\prime}$. This is because, if $\left(q, d^{\prime}\right)=1$,

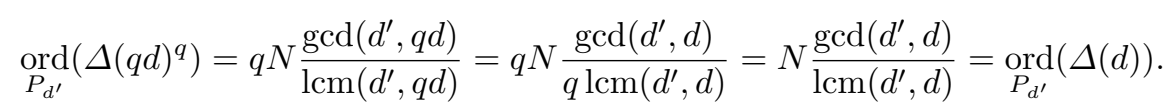

Similarly, the function

$$
\prod_{d \mid(N / q)}\left(\frac{\Delta(q d z)}{\Delta(d z)^{q}}\right)^{\mu(N / q d)}
$$

is supported on the set of cusps with $\left(q, d^{\prime}\right)=1$. Let $g_{d}(z)=\Delta(q d z) / \Delta(d z)^{q}$. Repeating the procedure with $f_{d}$ and $g_{d}$ for all primes $q \mid N, q \neq p_{0}$, we see that we end up with a product of simple functions. At every stage we have to multiply the divisor by $q+1$, which gives us our result.

\section{Remark 3.2.}

(1) The choice of the prime $p_{0}$ is arbitrary, so there are several different ways of decomposing a power of $\Delta_{N}$.

(2) This shows that one can find explicit annihilators of divisors of the form $P_{d}-P_{p_{0} d}$ for any prime $p_{0}$. Since the difference of any two cusps can be expressed as a sequence of such differences, one can use this to find annihilators of that and hence a 'new' proof of the Manin-Drinfel'd Theorem.

For example, if $N=p q$, the function

$$
\Delta_{p q}(z)=\frac{\Delta(p q z) \Delta(z)}{\Delta(p z) \Delta(q z)}
$$

can be factored as

$$
\Delta_{p q}(z)=\frac{\Delta(p q z)^{p}}{\Delta(q z)} \frac{\Delta(z)}{\Delta(p z)^{p}} \frac{\Delta(p q z)}{\Delta(q z)^{p}} \frac{\Delta(z)^{p}}{\Delta(p z)}
$$

with

$$
\operatorname{div}\left(\frac{\Delta(p q)^{p}}{\Delta(q)} \frac{\Delta(1)}{\Delta(p)^{p}}\right)=\left(q^{2}-1\right)(p-1)\left(P_{p q}-P_{q}\right)
$$

and

$$
\operatorname{div}\left(\frac{\Delta(p q)}{\Delta(q)^{p}} \frac{\Delta(1)^{p}}{\Delta(p)}\right)=\left(q^{2}-1\right)(p-1)\left(P_{1}-P_{p}\right)
$$




\subsubsection{An element of the motivic cohomology}

Let $\boldsymbol{\Delta}_{\mathbf{0}}(N)$ denote the diagonal on $X_{0}(N) \times X_{0}(N)$ and

$\Xi_{0}(N):=\left(\Delta_{\mathbf{0}}(N), \Delta_{N}^{\kappa}\right)-\left(\sum_{d \mid\left(N / p_{0}\right)}\left(X_{0}(N) \times P_{d}, F_{d} \times P_{d}\right)+\left(P_{d p_{0}} \times X_{0}(N), P_{d p_{0}} \times F_{d}\right)\right)$,

where by $F_{d} \times P_{d}$ we mean the function $F_{d}$ being considered as a function on $X_{0}(N) \times P_{d}$. This is an element of $H_{\mathcal{M}}^{3}\left(X_{0}(N) \times X_{0}(N), \mathbb{Q}(2)\right)$ as, from the decomposition above, the sum of the divisors is a sum of multiples of terms of the form

$$
\left(P_{d}, P_{d}\right)-\left(P_{d p_{0}}, P_{d p_{0}}\right)+\left(P_{d p_{0}}, P_{d}\right)-\left(P_{d}, P_{d}\right)+\left(P_{d p_{0}}, P_{d p_{0}}\right)-\left(P_{d p_{0}}, P_{d}\right),
$$

which cancel out.

\subsubsection{A mild generalization}

Let $N_{1}$ and $N_{2}$ be two square-free integers and let $N=\operatorname{lcm}\left(N_{1}, N_{2}\right)$ and let $M=$ $\operatorname{gcd}\left(N_{1}, N_{2}\right)$. Assume that $N / M \neq 1$. We now generalize this method to construct an element in $H_{\mathcal{M}}^{3}\left(X_{0}(N) \times X_{0}(N), \mathbb{Q}(2)\right)$ using the unit $\Delta_{N_{1}, N_{2}}$. We first observe

$$
\Delta_{N_{1}, N_{2}}(z)=\Delta_{(N / M)}(M z)
$$

where the function $\Delta_{(N / M)}$ is being thought of as a function on $X_{0}(N)$.

On $X_{0}(N)$ one has the Atkin-Lehner operators, which act on the cusps as follows [8]: for $d, a$ dividing $N$,

$$
w_{d}\left(P_{a}\right)=P_{\operatorname{lcm}(d, a) / \operatorname{gcd}(d, a)} .
$$

We have the following proposition.

Proposition 3.3. Let $N_{1}, N_{2}$ be square-free integers and let $N=\operatorname{lcm}\left(N_{1}, N_{2}\right)$ and let $M=\operatorname{gcd}\left(N_{1}, N_{2}\right)$. Assume that $N / M \neq 1$. Then

$$
\operatorname{div}\left(w_{M}^{*}\left(\Delta_{N / M}\right)\right)=\operatorname{div}\left(\Delta_{N_{1}, N_{2}}\right) .
$$

Proof. From (3.2), it suffices to prove that for any $a \mid N$ and $d \mid(N / M)$,

$$
\underset{P_{a}}{\operatorname{ord}} w_{M}^{*}(\Delta(d))=\underset{P_{a}}{\operatorname{ord}} \Delta(M d)
$$

This follows immediately from (3.1) and (3.3).

Let $\pi$ denote the degeneracy map $\pi: X_{0}(N) \rightarrow X_{0}((N / M))$. Define an element $\Xi_{0}\left(N_{1}, N_{2}\right)$ of $H_{\mathcal{M}}^{3}\left(X_{0}(N) \times X_{0}(N), \mathbb{Q}(2)\right)$ as follows:

$$
\Xi_{0}\left(N_{1}, N_{2}\right):=w_{M}^{*}\left(\pi^{*}\left(\Xi_{0}(N / M)\right)\right) .
$$

Note that, when $M=1$, this is the same as $\Xi_{0}(N)$. 


\subsection{Regulators}

The regulator map is a generalization of the cycle class map to the motivic cohomology groups. In our case, the regulator of an element of the group $H_{\mathcal{M}}^{3}(X, \mathbb{Q}(2))$ can be realized as a current on the space of $(1,1)$-forms. If $\Xi=\sum(\mathcal{C}, f)$ is an element of $H_{\mathcal{M}}^{3}(X, \mathbb{Q}(2))$ and $\omega$ is a $(1,1)$-form, the regulator is

$$
\left\langle r_{\mathcal{D}}(\Xi), \omega\right\rangle=\sum \frac{1}{2 \pi \mathrm{i}} \int_{C} \log |f| \omega .
$$

In particular, if $\omega=f \mathrm{~d} z \wedge \bar{g} \mathrm{~d} \bar{z}$, where $f$ and $g$ are modular forms of weight 2 for $\Gamma_{0}(N)$, we have

$$
\left\langle r_{\mathcal{D}}\left(\Xi_{0}\left(N_{1}, N_{2}\right)\right), \omega\right\rangle=\frac{1}{2 \pi \mathrm{i}} \int_{\Delta_{\mathbf{0}}(N)} \kappa \log \left|\Delta_{N_{1}, N_{2}}\right| \omega,
$$

as, since the form restricts to 0 , the integral on the vertical and horizontal curves $P_{d p_{0}} \times X_{0}(N)$ and $X_{0}(N) \times P_{d}$ vanish.

Comparing this with $(2.15)$ and observing that, since $N / M$ is square free, $\kappa=[\Gamma$ : $\left.\Gamma_{0}(N / M)\right]$, we have the following.

Lemma 3.4. If $f$ and $g$ are eigenforms of weight 2 for $\Gamma_{0}(N)$ and $f \neq g$, then one has

$$
L^{\prime}\left(H^{1}(E) \otimes H^{1}\left(E^{\prime}\right), 1\right)=\frac{-2 \pi^{2} \mathrm{i}}{3\left[\Gamma: \Gamma_{0}(N / M)\right]}\left\langle r_{\mathcal{D}}\left(\Xi_{0}\left(N_{1}, N_{2}\right)\right), \delta(f, g)\right\rangle .
$$

Combining this with (2.16) we have our next result.

Theorem 3.5. Let $E, E^{\prime}, f, g$ be as in (1.2). One has

$$
L^{\prime}\left(H^{1}(E) \otimes H^{1}\left(E^{\prime}\right), 1\right)=\frac{-\operatorname{deg}(\psi)}{12 c(\phi) c\left(\phi^{\prime}\right)\left[\Gamma: \Gamma_{0}(N / M)\right]}\left\langle r_{\mathcal{D}}\left(\psi_{*}\left(\Xi_{0}\left(N_{1}, N_{2}\right)\right)\right), \omega_{E} \wedge \bar{\omega}_{E^{\prime}}\right\rangle,
$$

where $\psi$ is the map from $X_{0}(N) \times X_{0}(N)$ to $E \times E^{\prime}$.

This formula is analogous to the formula (1.2) as it expresses the special value in terms of a regulator of an explicit element of the self-product of the modular parametrization. The product of the two non-isogenous elliptic curves is akin to the real quadratic field and the self-product of the modular parametrization is akin to the cyclotomic field containing the quadratic field.

Remark 3.6. Beilinson [2] shows that $c_{H^{1}(E) \otimes H^{1}\left(E^{\prime}\right)}(1)$ is the regulator of an element of the motivic cohomology, so part (iv) of his conjecture follows from this calculation.

\subsection{A 'class-number formula'}

To add credence to our claim that the element we have is an analogue of a cyclotomic unit, we show that there is a 'class-number formula' analogous to the expression (1.3) for $\zeta_{K}^{\prime}(0)$. 
We have the following curious product formula for our function $\Delta_{N}(z)$ which can be found in $[\mathbf{1}]$ :

$$
\Delta_{N_{1}, N_{2}}(z)=\sum_{d \mid(N / M)} \Delta\left(\frac{N z}{M d}\right)^{\mu(d)}=q^{\phi(N / M)} \prod_{n=1}^{\infty} \Phi_{(N / M)}\left(q^{n M}\right)^{24},
$$

where $\phi(N)$ is Euler's totient function, $\Phi_{N}(X)$ is the $N$ th cyclotomic polynomial, and $q=\mathrm{e}^{2 \pi \mathrm{i} z}$. This follows from the Möbius inversion formula applied to the identity

$$
\sum_{d \mid(N / M)} \log \Phi_{d}(X)=\log \left(1-X^{(N / M)}\right) .
$$

The inversion formula implies that

$$
\log \left(\Phi_{(N / M)}(X)\right)=\sum_{d \mid(N / M)} \mu(d) \log \left(1-X^{N / M d}\right) .
$$

We also have

$$
\Phi_{(N / M)}(X)=\prod_{\substack{k \bmod (N / M) \\(k,(N / M))=1}}\left(1-\xi^{k} X\right)
$$

where $\xi=\mathrm{e}^{M 2 \pi \mathrm{i} / N}$.

Define the $q$-logarithm for $q=\mathrm{e}^{2 \pi \mathrm{i} z}$ as in (1.4). Combining this with the formula for $L^{\prime}\left(H^{2}\left(E \times E^{\prime}\right), 1\right)$, we get the following result.

Theorem 3.7 (an 'elliptic class-number formula'). Let $E, E^{\prime}, f, g$ be as in (1.2). We have

$$
L^{\prime}\left(H^{1}(E) \otimes H^{1}\left(E^{\prime}\right), 1\right)=-\frac{1}{2} \sum_{\substack{k \bmod (N / M) \\(k, N / M)=1}} \frac{1}{2 \pi \mathrm{i}} \int_{X_{0}(N)} \log _{q}\left(\xi^{k}\right) f(q) \overline{g(q)} \frac{\mathrm{d} q}{q} \frac{\mathrm{d} \bar{q}}{\bar{q}}
$$

Proof. From (2.10) and (2.15) we have

$$
\begin{aligned}
L^{\prime}\left(H^{1}(E) \otimes H^{1}\left(E^{\prime}\right), 1\right) & =-4 \pi^{2} \mathrm{i} \frac{1}{2 \pi \mathrm{i}} \int_{X_{0}(N)} \phi((N / M)) \log |q| \delta(f, g) \\
& =-4 \pi^{2} \mathrm{i} \frac{1}{2 \pi \mathrm{i}} \int_{X_{0}(N)} \sum_{\substack { n=1 \\
\begin{subarray}{c}{k \bmod (N / M) \\
(k,(N / M))=1{ n = 1 \\
\begin{subarray} { c } { k \operatorname { m o d } ( N / M ) \\
( k , ( N / M ) ) = 1 } }\end{subarray}}^{\infty} \log \left|1-\xi^{k} q^{n}\right| \delta(f, g) .
\end{aligned}
$$

So the result follows from the definition of $\log _{q}(t)$. Note the similarity to (1.3).

In fact, a finer result holds. Up to a constant $C, \log _{q}\left(\xi^{k}\right)$ is simply the logarithm of a product of Siegel units $[\mathbf{5}], g_{\alpha, \beta}$ :

$$
\log _{q}\left(\xi^{k}\right)=C \log \left(\prod_{a \bmod N} g_{(a / N),(k / N)}(z)\right),
$$

which are certain modular units on $X_{1}(N)$. The divisor can be computed using the formula in $\left[\mathbf{5}\right.$, p. 40]. Using this one can construct elements of $H_{\mathcal{M}}^{3}\left(X_{1}(N) \times X_{1}(N), \mathbb{Q}(2)\right)$ which are direct analogues of cyclotomic units. 


\subsection{The case where $E^{\prime}$ is a twist of $E$}

This case - when $E$ is not isogenous over $\mathbb{Q}$ but is isogenous over an extension field, this necessarily being a quadratic extension - is interesting as it is half way between the other two cases. We assume the quadratic character $\chi$ has conductor $r$ prime to the level $N_{1}$ of $E$. Then the level of $E^{\prime}=E_{\chi}$ is $N_{2}=N_{1} r^{2}$. Let $\mathbb{Q}(\chi)$ be the fixed field of $\chi$. Since the corresponding modular forms $f$ and $f_{\chi}$ are newforms of different levels, their Petersson inner product is 0 , so $L_{f, f_{\chi}}(s)$ has a simple zero at 0 . This is also clear from the Tate conjecture as there are only two cycles in $B^{1}\left(E \times E_{\chi}\right)$ defined over $\mathbb{Q}$, the third cycle coming from the isogeny being only defined over $\mathbb{Q}(\chi)$.

The conjectures then imply that there is a non-trivial element of the motivic cohomology $H_{\mathcal{M}}^{3}\left(E \times E_{\chi}\right)$ whose regulator should equal the value of $L_{f, f_{\chi}}^{\prime}(0)$ up to some well-understood factors. Of course there is a cyclotomic element as above, but, in this case, as for the $L$-function factors, there is a simpler description, at least in the case when $\mathbb{Q}(\chi)$ is real quadratic.

The $L$-function factors as

$$
L_{f, f_{\chi}}(s)=L(s, \chi) L\left(\operatorname{Sym}^{2}(f) \otimes \chi, s+1\right)
$$

from which one has

$$
L_{f, f_{\chi}}^{\prime}(s)=L^{\prime}(s, \chi) L\left(\operatorname{Sym}^{2}(f) \otimes \chi, s+1\right)+L(s, \chi) L^{\prime}\left(\operatorname{Sym}^{2}(f) \otimes \chi, s+1\right) .
$$

At $s=0$, if $\chi$ is even, $L(0, \chi)=0$ and, from (1.3),

$$
L^{\prime}(0, \chi)=\log \prod_{\substack{k \bmod r \\(k, r)=1}}\left|1-\xi^{k}\right|^{-\chi(k) / 2}=\sum_{\substack{k \bmod N \\(k, N)=1}}-\frac{1}{2} \chi(k) \log \left|1-\xi^{k}\right|:=\log \left|U_{\chi}\right|,
$$

where $\xi=\mathrm{e}^{2 \pi \mathrm{i} / r}$. Furthermore, from $[\mathbf{3}]$ one has

$$
L\left(1, \operatorname{Sym}^{2}(f) \otimes \chi\right) \sim \mathbb{Q}^{*} \frac{\pi(f, f)}{g(\chi)},
$$

where $g(\chi)$ is the Gauss sum. Putting (3.6) and (3.8) together shows that

$$
L_{f, f_{\chi}}^{\prime}(0) \sim_{\mathbb{Q}^{*}} \log \left|U_{\chi}\right| \frac{\pi(f, f)}{g(\chi)} .
$$

This is nothing but the regulator of the decomposable element $\left(C_{\chi}, U_{\chi}\right)$, where $C_{\chi}$ is the graph of the isogeny and $U_{\chi}$ is the circular unit! This also shows that our classnumber formula can be thought of as a generalization of the usual class-number formula, as it specializes to the usual class-number formula in this case.

In the case of imaginary quadratic extensions, the zero comes from the twisted symmetric square $L$-function and not from the Dirichlet $L$-function of $\chi$. Here one has to understand the value of $L^{\prime}\left(1, \operatorname{Sym}^{2}(f) \otimes \chi\right)$. It appears in this case that the only way to compute it is in terms of the Beilinson elements, as the element does not decompose. 
Table 1. Correspondence between quadratic fields and elliptic curves

\begin{tabular}{ll}
\hline \multicolumn{1}{c}{ classical object } & elliptic analogue \\
\hline quadratic field $K$ & $E \times E^{\prime}$ \\
$\mathcal{O}_{K}^{*}=H_{\mathcal{M}}^{1}(\operatorname{Spec}(K) \mathbb{Q}(1))_{\mathbb{Z}}$ & $H_{\mathcal{M}}^{3}\left(E \times E^{\prime}, \mathbb{Q}(2)\right)_{\mathbb{Z}}$ \\
real quadratic $K$ & $E \times E^{\prime}$ non-isogenous \\
circular unit $U_{\chi}$ & element $\Xi_{0}\left(N_{1}, N_{2}\right)$ \\
$L^{\prime}(\chi, 0)$ & $L^{\prime}\left(H^{1}(E) \otimes H^{1}\left(E^{\prime}\right), 1\right)$ \\
cyclotomic units in $Q\left(\xi_{N}\right)$ & 'Siegel' elements in $X_{1}(N)^{2}$ \\
imaginary quadratic field & $E \times E^{\prime}$ isogenous \\
Gauss sum & Flach-Mildenhall elements \\
class group & Mildenhall's class group \\
$h_{K}$ & order of Mildenhall's group? \\
class-number formula & $?$ \\
\hline
\end{tabular}

\section{Epilogue or preface}

In Table 1 we summarize the analogy between products of elliptic curves and quadratic fields. While there appear to be analogues of every classical object, much less is known about the case of products of elliptic curves. For example, Mildenhall [8] defines a class group for products of elliptic curves which should be finite, but that is not known in general. He shows that the group is torsion in the case of $E \times E$, but nothing is known about any other case.

The analytic class-number formula (1.3) shows that the index of the group of circular units is the class number of the quadratic field. This is the starting point of the theory of circular units of Sinnott [13]. Perhaps there is a similar theory here. However, in this case there is no known analogue of Dirichlet class-number formula. Thaine $[\mathbf{1 4}]$ proved that the circular units give annihilators of the class group without using $L$-functions. Perhaps his methods could be applied to show that the Mildenhall class group is torsion or finite using the analogues of circular units here. It is likely that Kolyvagin's theory of Euler systems would have to be used.

Acknowledgements. The authors thank S. Bloch, E. Ghate, K. Rogale, M. Sundara and the referee for their comments. R.S. also thanks the Duke University Mathematics Department for its hospitality when this work was started. S.B. thanks Queens University for its hospitality and the Duke-IMRN conference for providing an opportunity for this work to be completed.

\section{References}

1. T. ASAI, On a certain modular function corresponding to a real cyclotomic field, in Seminar on Modern Methods in Number Theory, Institute of Statistics and Mathematics, Tokyo, 1971, Paper 9 (Institute of Statistics and Mathematics of Tokyo, 1971).

2. A. A. Beilinson, Higher regulators and values of L-functions, Current Problems in Mathematics, vol. 24, pp. 181-238 (VINITI, Moscow, 1984) (in Russian). 
3. J. CoAtes And C.-G. SChimdt, Iwasawa theory for the symmetric square of an elliptic curve, J. Reine Angew. Math. 375/376 (1987), 104-156.

4. P. Deligne, Valeurs de fonctions $L$ et periodes d'integrales, With an appendix by N. Koblitz and A. Ogus, in Proc. Symp. Pure Mathematics, XXXIII, Automorphic Forms, Representations and L-functions, Oregon State University, Corvallis, OR, 197r, Part 2, pp. 313-346 (American Mathematical Society, Providence, RI, 1979) (in French) (English transl. E. Ghate and J. Beineke, http://www.math.tifr.res.in/ eghate).

5. D. KUBert AND S. LANG, Modular units, Grundlehren der Mathematischen Wissenschaften (Fundamental Principles of Mathematical Science), vol. 244 (Springer, 1981).

6. S. LANG, Elliptic functions, with an appendix by J. Tate, 2nd edn, Graduate Texts in Mathematics, vol. 112 (Springer, 1987).

7. W. C. W. LI, L-series of Rankin type and their functional equations, Math. Ann. 244 (1979), 135-166.

8. S. J. M. Mildenhall, Cycles in a product of elliptic curves, and a group analogous to the class group, Duke Math. J. 67 (1992), 387-406.

9. M. Newman, Construction and application of a class of modular functions, II, Proc. Lond. Math. Soc. 9 (1959), 373-387.

10. A. P. OGG, On a convolution of L-series, Invent. Math. 7 (1969), 297-312.

11. P. SCHNEIDER, Introduction to the Beilinson conjectures, in Beilinson's conjectures on special values of L-functions, pp. 1-35, Perspectives in Mathematics, vol. 4 (Academic, 1988).

12. A. J. ScholL, Integral elements of $K$-theory and products of modular curves, The Arithmetic and Geometry of Algebraic Cycles, NATO Science Series, vol. 548, pp. 467-489 (Kluwer, 2000).

13. W. Sinnott, On the Stickelberger ideal and the circular units of an abelian field, Invent. Math. 62 (1980), 181-234.

14. F. Thaine, On the ideal class groups of real abelian number fields, Ann. Math. 128 (1988), 1-18. 